\title{
Binomial difference sequence spaces of fractional order
}

\author{
Jian Meng ${ }^{1}$ and Liquan Mei ${ }^{1^{*}}$
}

\section{"Correspondence: \\ lqmei@mail.xjtu.edu.cn \\ ${ }^{1}$ School of Mathematics and \\ Statistics, Xi'an Jiaotong University, \\ Xi'an, P.R. China}

\begin{abstract}
In this paper, we introduce the sequence spaces $b_{0}^{r, s}\left(\nabla^{(\alpha)}\right), b_{c}^{r, s}\left(\nabla^{(\alpha)}\right)$, and $b_{\infty}^{r, s}\left(\nabla^{(\alpha)}\right)$. We investigate some functional properties, inclusion relations, and the $\alpha-, \beta-, \gamma-$, and continuous duals of these sets.

MSC: 46A45; 46B45; 46B50

Keywords: Sequence space; Matrix transformation; Fractional difference operator; $\alpha-, \beta$-, and $\gamma$-duals
\end{abstract}

\section{Introduction}

Let $w, \ell_{p}, \ell_{\infty}, c$, and $c_{0}$ denote the spaces of all, $p$-absolutely summable, bounded, convergent, and null sequences $x=\left(x_{k}\right)$ with complex terms $x_{k}$, respectively, where $1 \leq p<\infty$ and $k \in \mathbb{N}=\{0,1,2, \ldots\}$. A sequence space $X$ is called a $B K$-space if it is a Banach space with continuous coordinates $p_{k}: X \rightarrow \mathbb{C}$ defined by $p_{k}(x)=x_{k}$ for $x=\left(x_{k}\right) \in X$ and $k \in \mathbb{N}$. The most important result of the theory of $B K$-spaces is that matrix mappings between $B K$-spaces are continuous [13]. The sequence spaces $\ell_{\infty}, c$, and $c_{0}$ with their sup-norm are $B K$-spaces.

The concept of a difference sequence space was firstly introduced by Kizmaz [22] by defining the set $Z(\Delta)=\left\{x=\left(x_{k}\right):\left(\Delta x_{k}\right) \in Z\right\}$ for $Z \in\left\{\ell_{\infty}, c, c_{0}\right\}$, where $\Delta x_{k}=x_{k}-x_{k+1}$ for $k \in \mathbb{N}$. The idea of a difference sequence was generalized by Et and Çolak [14-16] by defining the spaces $Z\left(\Delta^{m}\right)=\left\{x=\left(x_{k}\right):\left(\Delta^{m} x_{k}\right) \in Z\right\}$ for $Z \in\left\{\ell_{\infty}, c, c_{0}\right\}$, where $m \in \mathbb{N}$, $\Delta^{m} x_{k}=\Delta^{m-1} x_{k}-\Delta^{m-1} x_{k+1}$ for $k \in \mathbb{N}$. For a positive proper fraction $\alpha$, Baliarsingh and Dutta $[4,5]$ defined the fractional difference operator $\Delta^{\alpha}$ by

$$
\Delta^{\alpha} x_{k}=\sum_{i=0}^{\infty}(-1)^{i} \frac{\Gamma(\alpha+1)}{i ! \Gamma(\alpha-i+1)} x_{k+i}
$$

for $k \in \mathbb{N}$, where the Euler gamma function $\Gamma(p)$ of a real number $p$ with $p \notin\{0,-1,-2$, $-3, \ldots\}$ can be expressed as an improper integral $\Gamma(p)=\int_{0}^{\infty} e^{-t} t^{p-1} d t$. It is observed that

(i) $\Gamma(p+1)=p$ ! for $p \in \mathbb{N}$,

(ii) $\Gamma(p+1)=p \Gamma(p)$ for $p \in \mathbb{R} \backslash\{0,-1,-2,-3, \ldots\}$.

Some definitions of fractional derivatives have been generalized by using a set of new difference sequence spaces of fractional order [3]. Application of fractional derivatives

(c) The Author(s) 2018. This article is distributed under the terms of the Creative Commons Attribution 4.0 International License (http://creativecommons.org/licenses/by/4.0/), which permits unrestricted use, distribution, and reproduction in any medium, provided you give appropriate credit to the original author(s) and the source, provide a link to the Creative Commons license, and indicate if changes were made. 
becomes more apparent in diffusion processes, modeling mechanical systems, and many other fields.

Let $X, Y$ be two sequence spaces, and let $A=\left(a_{n, k}\right)$ be an infinite matrix with complex numbers $a_{n, k}, n, k \in \mathbb{N}$. Let the sequence space $X_{A}$ defined by $X_{A}=\left\{x=\left(x_{k}\right): A x \in X\right\}$ denote the domain of matrix $A$ in the space $X$, where $A x=\left\{(A x)_{n}\right\}$, the $A$-transform of $x$, is defined by $(A x)_{n}=\sum_{k=0}^{\infty} a_{n, k} x_{k}, n \in \mathbb{N}$. Let $(X: Y)$ denote the class of all matrices such that $X \subseteq Y_{A}$. The matrix domain approach has been employed by Başarir and Kara [610], Kara and Illkhan [19-21], Polat and Başar [26], Song and Meng [23-25, 27], and many others to introduce new sequence spaces.

The Euler sequence spaces $e_{0}^{r}, e_{c}^{r}$, and $e_{\infty}^{r}$ were defined by Altay and Başar [1] and Altay, Başar, and Mursaleen [2]. Moreover, Kadak and Baliarsingh [18] introduced a generalized Euler mean difference operator $E^{r}\left(\nabla^{(\alpha)}\right)$ of fractional order, where the matrix $\nabla^{(\alpha)}=\left(\nabla_{n, k}^{(\alpha)}\right)$ is defined by

$$
\nabla_{n, k}^{(\alpha)}= \begin{cases}(-1)^{n-k} \frac{\Gamma(\alpha+1)}{(n-k) ! \Gamma(\alpha-n+k+1)} & \text { if } 0 \leq k \leq n, \\ 0 & \text { if } k>n\end{cases}
$$

Let $r, s \in \mathbb{R}$ and $r+s \neq 0$. Then the binomial matrix $B^{r, s}=\left(b_{n, k}^{r, s}\right)$ is defined by

$$
b_{n, k}^{r, s}= \begin{cases}\frac{1}{(s+r)^{n}}\left(\begin{array}{l}
n \\
k
\end{array}\right) s^{n-k} r^{k} & \text { if } 0 \leq k \leq n \\
0 & \text { if } k>n,\end{cases}
$$

for all $k, n \in \mathbb{N}$. If $s+r=1$, then we obtain the Euler matrix $E^{r}$. Bişgin $[11,12]$ defined the binomial sequence spaces

$$
\begin{aligned}
& b_{0}^{r, s}=\left\{x=\left(x_{k}\right): \lim _{n \rightarrow \infty} \frac{1}{(s+r)^{n}} \sum_{k=0}^{n}\left(\begin{array}{l}
n \\
k
\end{array}\right) s^{n-k} r^{k} x_{k}=0\right\}, \\
& b_{c}^{r, s}=\left\{x=\left(x_{k}\right): \lim _{n \rightarrow \infty} \frac{1}{(s+r)^{n}} \sum_{k=0}^{n}\left(\begin{array}{l}
n \\
k
\end{array}\right) s^{n-k} r^{k} x_{k} \text { exists }\right\},
\end{aligned}
$$

and

$$
b_{\infty}^{r, s}=\left\{x=\left(x_{k}\right): \sup _{n \in \mathbb{N}}\left|\frac{1}{(s+r)^{n}} \sum_{k=0}^{n}\left(\begin{array}{l}
n \\
k
\end{array}\right) s^{n-k} r^{k} x_{k}\right|<\infty\right\} .
$$

The purpose of this paper is to generalize the sequence spaces $e_{0}^{r}\left(\nabla^{(\alpha)}\right), e_{c}^{r}\left(\nabla^{(\alpha)}\right)$, and $e_{\infty}^{r}\left(\nabla^{(\alpha)}\right)$ and introduce the binomial difference sequence spaces $b_{0}^{r, s}\left(\nabla^{(\alpha)}\right), b_{c}^{r, s}\left(\nabla^{(\alpha)}\right)$, and $b_{\infty}^{r, s}\left(\nabla^{(\alpha)}\right)$ of fractional order whose $\nabla^{(\alpha)}$-transforms are in the spaces $b_{0}^{r, s}, b_{c}^{r, s}$, and $b_{\infty}^{r, s}$. These new sequence spaces are generalizations of the spaces defined in $[11,12,18,23,25$, 26].

\section{Difference sequence spaces of fractional order}

In this chapter, we introduce the binomial difference sequence spaces $b_{0}^{r, s}\left(\nabla^{(\alpha)}\right), b_{c}^{r, s}\left(\nabla^{(\alpha)}\right)$, and $b_{\infty}^{r, s}\left(\nabla^{(\alpha)}\right)$ of fractional order and investigate some functional properties and inclusion relations. 
Define the spaces $b_{0}^{r, s}\left(\nabla^{(\alpha)}\right), b_{c}^{r, s}\left(\nabla^{(\alpha)}\right)$, and $b_{\infty}^{r, s}\left(\nabla^{(\alpha)}\right)$ by

$$
Z\left(\nabla^{(\alpha)}\right)=\left\{x=\left(x_{k}\right): \nabla^{(\alpha)}\left(x_{k}\right) \in Z\right\}
$$

for $Z \in\left\{b_{0}^{r, s}, b_{c}^{r, s}, b_{\infty}^{r, s}\right\}$. By taking the $\nabla^{(\alpha)}$-transform of $x$ in the spaces $b_{0}^{r, s}, b_{c}^{r, s}$, and $b_{\infty}^{r, s}$ the spaces $b_{0}^{r, s}\left(\nabla^{(\alpha)}\right), b_{c}^{r, s}\left(\nabla^{(\alpha)}\right)$, and $b_{\infty}^{r, s}\left(\nabla^{(\alpha)}\right)$ can be redefined by

$$
b_{0}^{r, s}\left(\nabla^{(\alpha)}\right)=\left(b_{0}^{r, s}\right)_{\nabla^{(\alpha)}}, \quad b_{c}^{r, s}\left(\nabla^{(\alpha)}\right)=\left(b_{c}^{r, s}\right)_{\nabla^{(\alpha)}}, \quad b_{\infty}^{r, s}\left(\nabla^{(\alpha)}\right)=\left(b_{\infty}^{r, s}\right)_{\nabla^{(\alpha)}} .
$$

The sequence spaces $b_{0}^{r, s}\left(\nabla^{(\alpha)}\right), b_{c}^{r, s}\left(\nabla^{(\alpha)}\right)$, and $b_{\infty}^{r, s}\left(\nabla^{(\alpha)}\right)$ include some particular cases in certain cases of $s, r$, and $\alpha$.

(i) For $\alpha=0$, these sequence spaces generalize the spaces $b_{0}^{r, s}, b_{c}^{r, s}$, and $b_{\infty}^{r, s}$ defined by Bişgin $[11,12]$.

(ii) For $s+r=1$, these sequence spaces generalize the spaces $e_{0}^{r}\left(\nabla^{(\alpha)}\right), e_{c}^{r}\left(\nabla^{(\alpha)}\right)$, and $e_{\infty}^{r}\left(\nabla^{(\alpha)}\right)$ defined by Kadak and Baliarsingh [18].

(iii) For $\alpha=m \in \mathbb{N}$, these sequence spaces generalize the spaces $b_{0}^{r, s}\left(\nabla^{(m)}\right), b_{c}^{r, s}\left(\nabla^{(m)}\right)$, and $b_{\infty}^{r, s}\left(\nabla^{(m)}\right)$ defined by Meng and Song [23].

(iv) For $s+r=1$ and $\alpha=m \in \mathbb{N}$, these sequence spaces generalize the spaces $e_{0}^{r}\left(\nabla^{(m)}\right)$, $e_{c}^{r}\left(\nabla^{(m)}\right)$, and $e_{\infty}^{r}\left(\nabla^{(m)}\right)$ defined by Polat and Başar [26].

Define the sequence $y=\left(y_{n}\right)$ by the $B^{r, s}\left(\nabla^{(\alpha)}\right)$-transform of a sequence $x=\left(x_{k}\right)$, that is,

$$
\begin{aligned}
y_{n} & =\left[B^{r, s}\left(\nabla^{(\alpha)}\right)\left(x_{k}\right)\right]_{n} \\
& =\frac{1}{(s+r)^{n}} \sum_{k=0}^{n} \sum_{i=k}^{n}(-1)^{i-k}\left(\begin{array}{l}
n \\
i
\end{array}\right) \frac{\Gamma(\alpha+1)}{(i-k) ! \Gamma(\alpha-i+k+1)} s^{n-i} r^{i} x_{k}
\end{aligned}
$$

for each $n \in \mathbb{N}$.

Theorem 2.1 Let $Z \in\left\{b_{0}^{r, s}, b_{c}^{r, s}, b_{\infty}^{r, s}\right\}$. Then $Z\left(\nabla^{(\alpha)}\right)$ are BK-spaces with the norm $\|x\|_{Z\left(\nabla^{(\alpha)}\right)}=$ $\left\|\nabla^{(\alpha)}\left(x_{k}\right)\right\|_{Z}$.

Proof Theorem 2.1 of Bişgin [11, 12] and Theorem 4.3.12 of Wilansky [29] imply that the spaces $Z\left(\nabla^{(\alpha)}\right)$ are $B K$-spaces.

Theorem 2.2 The inclusion $b_{0}^{r, s}\left(\nabla^{(\alpha)}\right) \subseteq b_{c}^{r, s}\left(\nabla^{(\alpha)}\right) \subseteq b_{\infty}^{r, s}\left(\nabla^{(\alpha)}\right)$ is strict.

Proof Proof follows from Lemma 2.3 of Et and Nuray [17].

Theorem 2.3 The inclusions $e_{0}^{r}\left(\nabla^{(\alpha)}\right) \subseteq b_{0}^{r, s}\left(\nabla^{(\alpha)}\right), e_{c}^{r}\left(\nabla^{(\alpha)}\right) \subseteq b_{c}^{r, s}\left(\nabla^{(\alpha)}\right)$, and $e_{\infty}^{r}\left(\nabla^{(\alpha)}\right) \subseteq$ $b_{\infty}^{r, s}\left(\nabla^{(\alpha)}\right)$ are strict.

Proof We only give the proof of the inclusion $e_{0}^{r}\left(\nabla^{(\alpha)}\right) \subseteq b_{0}^{r, s}\left(\nabla^{(\alpha)}\right)$. The others can be proved similarly.

It is clear that $e_{0}^{r}\left(\nabla^{(\alpha)}\right) \subseteq b_{0}^{r, s}\left(\nabla^{(\alpha)}\right)$. Further, to show that this inclusion is strict, let $0<$ $r<1$ and $s=4$ and define the sequence $x=\left(x_{k}\right)$ by

$$
x_{k}=\sum_{j=0}^{k}(-1)^{k-j} \frac{\Gamma(-\alpha+1)}{(k-j) ! \Gamma(-\alpha-k+j+1)}\left(-\frac{3}{r}\right)^{j}
$$


for $k \in \mathbb{N}$. We have $\left[E^{r}\left(\nabla^{(\alpha)}\right)\left(x_{k}\right)\right]_{n}=\left((-2-r)^{n}\right) \notin c_{0}$ and $\left[B^{r, s}\left(\nabla^{(\alpha)}\right)\left(x_{k}\right)\right]_{n}=\left(\left(\frac{1}{4+r}\right)^{n}\right) \in c_{0}$. Therefore, the inclusion $e_{0}^{r}\left(\nabla^{(\alpha)}\right) \subseteq b_{0}^{r, s}\left(\nabla^{(\alpha)}\right)$ is strict.

Theorem 2.4 The spaces $b_{0}^{r, s}\left(\nabla^{(\alpha)}\right), b_{c}^{r, s}\left(\nabla^{(\alpha)}\right)$, and $b_{\infty}^{r, s}\left(\nabla^{(\alpha)}\right)$ are linearly isomorphic to the spaces $c_{0}, c$, and $\ell_{\infty}$, respectively.

Proof We prove the theorem only for the space $b_{0}^{r, s}\left(\nabla^{(\alpha)}\right)$. To prove $b_{0}^{r, s}\left(\nabla^{(\alpha)}\right) \cong c_{0}$, we will show the existence of a linear bijection between the spaces $b_{0}^{r, s}\left(\nabla^{(\alpha)}\right)$ and $c_{0}$.

Let us denote the transformation $T: b_{0}^{r, s}\left(\nabla^{(\alpha)}\right) \rightarrow c_{0}$ by $T(x)=B^{r, s}\left(\nabla^{(\alpha)}\right)\left(x_{k}\right)$. The linearity of $T$ is clear, and $x=0$ whenever $T(x)=0$. Hence $T$ is injective.

Let $y=\left(y_{n}\right) \in c_{0}$ and define the sequence $x=\left(x_{k}\right)$ by

$$
x_{k}=\sum_{i=0}^{k}(s+r)^{i} \sum_{j=i}^{k}(-1)^{k-j}\left(\begin{array}{l}
j \\
i
\end{array}\right) \frac{\Gamma(-\alpha+1)}{(k-j) ! \Gamma(-\alpha-k+j+1)} r^{-j}(-s)^{j-i} y_{i}
$$

for $k \in \mathbb{N}$. Then we have

$$
\lim _{n \rightarrow \infty}\left[B^{r, s}\left(\nabla^{(\alpha)}\right)\left(x_{k}\right)\right]_{n}=\lim _{n \rightarrow \infty} \frac{1}{(s+r)^{n}} \sum_{k=0}^{n}\left(\begin{array}{l}
n \\
k
\end{array}\right) s^{n-k} r^{k}\left(\nabla^{(\alpha)}\right)\left(x_{k}\right)=\lim _{n \rightarrow \infty} y_{n}=0,
$$

which implies that $x \in b_{0}^{r, s}\left(\nabla^{(\alpha)}\right)$. Therefore, we obtain that $T$ is surjective and norm preserving. This completes the proof.

We shall construct the Schauder bases for the sequence spaces $b_{0}^{r, s}\left(\nabla^{(\alpha)}\right)$ and $b_{c}^{r, s}\left(\nabla^{(\alpha)}\right)$. Because the isomorphism $T$ between $b_{0}^{r, s}\left(\nabla^{(\alpha)}\right)$ and $c_{0}$ (or between $b_{c}^{r, s}\left(\nabla^{(\alpha)}\right)$ and $c$ ) is onto, the inverse image of the basis of the space $c_{0}$ (or $c$ ) is the basis of the space $b_{0}^{r, s}\left(\nabla^{(\alpha)}\right.$ ) (or $\left.b_{c}^{r, s}\left(\nabla^{(\alpha)}\right)\right)$. For $k \in \mathbb{N}$, define the sequence $g^{(k)}(r, s)=\left\{g_{i}^{(k)}(r, s)\right\}_{i \in \mathbb{N}}$ by

$$
g_{i}^{(k)}(r, s)= \begin{cases}0 & \text { if } 0 \leq i<k, \\
(s+r)^{k} \sum_{j=k}^{i}(-1)^{i-j}\left(\begin{array}{l}
j \\
k
\end{array}\right) \frac{\Gamma(-\alpha+1)}{(i-j) ! \Gamma(-\alpha-i+j+1)} r^{-j}(-s)^{j-k} & \text { if } i \geq k .\end{cases}
$$

Theorem 2.5 The sequence $\left(g^{(k)}(r, s)\right)_{k \in \mathbb{N}}$ is the Schauder basis for the space $b_{0}^{r, s}\left(\nabla^{(\alpha)}\right)$, and every $x$ in $b_{0}^{r, s}\left(\nabla^{(\alpha)}\right)$ has a unique representation by

$$
x=\sum_{k} \lambda_{k}(r, s) g^{(k)}(r, s)
$$

where $\lambda_{k}(r, s)=\left[B^{r, s}\left(\nabla^{(\alpha)}\right)\left(x_{i}\right)\right]_{k}$ for $k \in \mathbb{N}$.

Theorem 2.6 Define the sequence $g=\left(g_{n}\right)$ by

$$
g_{n}=\sum_{k=0}^{n}(s+r)^{k} \sum_{j=k}^{n}(-1)^{n-j}\left(\begin{array}{l}
j \\
k
\end{array}\right) \frac{\Gamma(-\alpha+1)}{(n-j) ! \Gamma(-\alpha-n+j+1)} r^{-j}(-s)^{j-k}
$$

for $n \in \mathbb{N}$ and $\lim _{k \rightarrow \infty} \lambda_{k}(r, s)=l$. The set $\left\{g, g^{(0)}(r, s), g^{(1)}(r, s), g^{(2)}(r, s), \ldots\right\}$ is the Schauder basis for the space $b_{c}^{r, s}\left(\nabla^{(\alpha)}\right)$, and every $x$ in $b_{c}^{r, s}\left(\nabla^{(\alpha)}\right)$ has a unique representation by

$$
x=l g+\sum_{k}\left[\lambda_{k}(r, s)-l\right] g^{(k)}(r, s) .
$$




\section{The $\alpha-, \beta-, \gamma-$, and continuous duals}

In this section, we determine the $\alpha-, \beta-, \gamma-$, and continuous duals of the spaces $b_{0}^{r, s}\left(\nabla^{(\alpha)}\right)$, $b_{c}^{r, s}\left(\nabla^{(\alpha)}\right)$, and $b_{\infty}^{r, s}\left(\nabla^{(\alpha)}\right)$.

For two sequence spaces $X$ and $Y$, the set $M(X, Y)$ is defined by

$$
M(X, Y)=\left\{u=\left(u_{k}\right): u x=\left(u_{k} x_{k}\right) \in Y \text { for all } x=\left(x_{k}\right) \in X\right\}
$$

Let $b s$ and $c s$ denote the sequence spaces of all bounded and convergent series, respectively. In particular,

$$
X^{\alpha}=M\left(X, \ell_{1}\right), \quad X^{\beta}=M(X, c s), \quad \text { and } \quad X^{\gamma}=M(X, b s)
$$

are called the $\alpha-, \beta$-, and $\gamma$-duals of the sequence space $X$, respectively. The space of all bounded linear functionals on $X$ denoted by $X^{*}$ is called the continuous dual of the space $X$.

Let us give the following properties needed in Lemma 3.1:

$$
\begin{aligned}
& \sup _{K \in \Gamma} \sum_{n}\left|\sum_{k \in K} a_{n, k}\right|<\infty, \\
& \sup _{n \in \mathbb{N}} \sum_{k}\left|a_{n, k}\right|<\infty, \\
& \lim _{n \rightarrow \infty} a_{n, k}=a_{k} \quad \text { for } k \in \mathbb{N}, \\
& \lim _{n \rightarrow \infty} \sum_{k} a_{n, k}=a, \\
& \lim _{n \rightarrow \infty} \sum_{k}\left|a_{n, k}\right|=\sum_{k}\left|\lim _{n \rightarrow \infty} a_{n, k}\right|,
\end{aligned}
$$

where $\Gamma$ is the collection of all finite subsets of $\mathbb{N}$.

Lemma 3.1 ([28]) Let $A=\left(a_{n, k}\right)$ be an infinite matrix. Then

(i) $A \in\left(c_{0}: \ell_{1}\right)=\left(c: \ell_{1}\right)=\left(\ell_{\infty}: \ell_{1}\right)$ if and only if (3.1) holds.

(ii) $A \in\left(c_{0}: c\right)$ if and only if (3.2) and (3.3) hold.

(iii) $A \in(c: c)$ if and only if (3.2), (3.3), and (3.4) hold.

(iv) $A \in\left(\ell_{\infty}: c\right)$ if and only if (3.3) and (3.5) hold.

(v) $A \in\left(c_{0}: \ell_{\infty}\right)=\left(c: \ell_{\infty}\right)=\left(\ell_{\infty}: \ell_{\infty}\right)$ if and only if (3.2) holds.

Theorem 3.2 We have $\left[b_{0}^{r, s}\left(\nabla^{(\alpha)}\right)\right]^{\alpha}=\left[b_{c}^{r, s}\left(\nabla^{(\alpha)}\right)\right]^{\alpha}=\left[b_{\infty}^{r, s}\left(\nabla^{(\alpha)}\right)\right]^{\alpha}=U_{1}^{r, s}$, where

$$
\begin{aligned}
U_{1}^{r, s}= & \left\{u=\left(u_{k}\right): \sup _{K \in \Gamma} \sum_{k} \mid \sum_{i \in K}(s+r)^{i} \sum_{j=i}^{k}(-1)^{k-j}\left(\begin{array}{l}
j \\
i
\end{array}\right)\right. \\
& \left.\times \frac{\Gamma(-\alpha+1)}{(k-j) ! \Gamma(-\alpha-k+j+1)} r^{-j}(-s)^{j-i} u_{k} \mid<\infty\right\} .
\end{aligned}
$$


Proof We immediately derive by (2.3) that

$$
u_{k} x_{k}=\sum_{i=0}^{k}(s+r)^{i} \sum_{j=i}^{k}(-1)^{k-j}\left(\begin{array}{l}
j \\
i
\end{array}\right) \frac{\Gamma(-\alpha+1)}{(k-j) ! \Gamma(-\alpha-k+j+1)} r^{-j}(-s)^{j-i} u_{k} y_{i}=\left(G^{r, s} y\right)_{k}
$$

for $k \in \mathbb{N}$, where $G^{r, s}=\left(g_{k, i}^{r, s}\right)$ is defined by

$$
g_{k, i}^{r, s}= \begin{cases}(s+r)^{i} \sum_{j=i}^{k}(-1)^{k-j}\left(\begin{array}{c}
j \\
i
\end{array}\right) \frac{\Gamma(-\alpha+1)}{(k-j) ! \Gamma(-\alpha-k+j+1)} r^{-j}(-s)^{j-i} u_{k} & \text { if } 0 \leq i \leq k \\
0 & \text { if } i>k\end{cases}
$$

Therefore $u x=\left(u_{k} x_{k}\right) \in \ell_{1}$ whenever $x \in b_{0}^{r, s}\left(\nabla^{(\alpha)}\right), b_{c}^{r, s}\left(\nabla^{(\alpha)}\right)$ or $b_{\infty}^{r, s}\left(\nabla^{(\alpha)}\right)$ if and only if $G^{r, s} y \in \ell_{1}$ whenever $y \in c_{0}, c$ or $\ell_{\infty}$. This implies that $u=\left(u_{k}\right) \in\left[b_{0}^{r, s}\left(\nabla^{(\alpha)}\right)\right]^{\alpha},\left[b_{c}^{r, s}\left(\nabla^{(\alpha)}\right)\right]^{\alpha}$, or $\left[b_{\infty}^{r, s}\left(\nabla^{(\alpha)}\right)\right]^{\alpha}$ if and only if $G^{r, s} \in\left(c_{0}: \ell_{1}\right)=\left(c: \ell_{1}\right)=\left(\ell_{\infty}: \ell_{1}\right)$. We derive by part (i) of Lemma 3.1 that $u=\left(u_{k}\right) \in\left[b_{0}^{r, s}\left(\nabla^{(\alpha)}\right)\right]^{\alpha}=\left[b_{c}^{r, s}\left(\nabla^{(\alpha)}\right)\right]^{\alpha}=\left[b_{\infty}^{r, s}\left(\nabla^{(\alpha)}\right)\right]^{\alpha}$ if and only if

$$
\sup _{K \in \Gamma} \sum_{k}\left|\sum_{i \in K}(s+r)^{i} \sum_{j=i}^{k}(-1)^{k-j}\left(\begin{array}{l}
j \\
i
\end{array}\right) \frac{\Gamma(-\alpha+1)}{(k-j) ! \Gamma(-\alpha-k+j+1)} r^{-j}(-s)^{j-i} u_{k}\right|<\infty
$$

which yields that $\left[b_{0}^{r, s}\left(\nabla^{(\alpha)}\right)\right]^{\alpha}=\left[b_{c}^{r, s}\left(\nabla^{(\alpha)}\right)\right]^{\alpha}=\left[b_{\infty}^{r, s}\left(\nabla^{(\alpha)}\right)\right]^{\alpha}=U_{1}^{r, s}$.

To determine the $\beta$ - and $\gamma$-duals of the spaces $b_{0}^{r, s}\left(\nabla^{(\alpha)}\right), b_{c}^{r, s}\left(\nabla^{(\alpha)}\right)$, and $b_{\infty}^{r, s}\left(\nabla^{(\alpha)}\right)$, we define the following sets:

$$
\begin{aligned}
& U_{2}^{r, s}=\left\{u=\left(u_{k}\right): \sup _{n \in \mathbb{N}} \sum_{k}\left|u_{n, k}\right|<\infty\right\}, \\
& U_{3}^{r, s}=\left\{u=\left(u_{k}\right): \lim _{n \rightarrow \infty} u_{n, k} \text { exists for each } k \in \mathbb{N}\right\}, \\
& U_{4}^{r, s}=\left\{u=\left(u_{k}\right): \lim _{n \rightarrow \infty} \sum_{k}\left|u_{n, k}\right|=\sum_{k}\left|\lim _{n \rightarrow \infty} u_{n, k}\right|\right\},
\end{aligned}
$$

and

$$
U_{5}^{r, s}=\left\{u=\left(u_{k}\right): \lim _{n \rightarrow \infty} \sum_{k} u_{n, k} \text { exists }\right\}
$$

where

$$
u_{n, k}=(s+r)^{k} \sum_{i=k}^{n} \sum_{j=k}^{i}(-1)^{i-j}\left(\begin{array}{l}
j \\
k
\end{array}\right) \frac{\Gamma(-\alpha+1)}{(i-j) ! \Gamma(-\alpha-i+j+1)} r^{-j}(-s)^{j-k} u_{i}
$$

\section{Theorem 3.3}

(i) $\left[b_{0}^{r, s}\left(\nabla^{(\alpha)}\right)\right]^{\beta}=U_{2}^{r, s} \cap U_{3}^{r, s}$,

(ii) $\left[b_{c}^{r, s}\left(\nabla^{(\alpha)}\right)\right]^{\beta}=U_{2}^{r, s} \cap U_{3}^{r, s} \cap U_{5}^{r, s}$,

(iii) $\left[b_{\infty}^{r, s}\left(\nabla^{(\alpha)}\right)\right]^{\beta}=U_{3}^{r, s} \cap U_{4}^{r, s}$,

(iv) $\left[b_{0}^{r, s}\left(\nabla^{(\alpha)}\right)\right]^{\gamma}=\left[b_{c}^{r, s}\left(\nabla^{(\alpha)}\right)\right]^{\gamma}=\left[b_{\infty}^{r, s}\left(\nabla^{(\alpha)}\right)\right]^{\gamma}=U_{2}^{r, s}$. 
Proof We consider the equality

$$
\begin{aligned}
\sum_{k=0}^{n} u_{k} x_{k} & =\sum_{k=0}^{n} u_{k}\left[\sum_{i=0}^{k}(s+r)^{i} \sum_{j=i}^{k}(-1)^{k-j}\left(\begin{array}{l}
j \\
i
\end{array}\right) \frac{\Gamma(-\alpha+1)}{(k-j) ! \Gamma(-\alpha-k+j+1)} r^{-j}(-s)^{j-i} y_{i}\right] \\
& =\sum_{k=0}^{n}\left[(s+r)^{k} \sum_{i=k}^{n} \sum_{j=k}^{i}(-1)^{i-j}\left(\begin{array}{l}
j \\
k
\end{array}\right) \frac{\Gamma(-\alpha+1)}{(i-j) ! \Gamma(-\alpha-i+j+1)} r^{-j}(-s)^{j-k} u_{i}\right] y_{k} \\
& =\left(U^{r, s} y\right)_{n},
\end{aligned}
$$

where $U^{r, s}=\left(u_{n, k}^{r, s}\right)$ is defined by

$$
u_{n, k}^{r, s}= \begin{cases}(s+r)^{k} \sum_{i=k}^{n} \sum_{j=k}^{i}(-1)^{i-j}\left(\begin{array}{c}
j \\
k
\end{array}\right) \frac{\Gamma(-\alpha+1)}{(i-j) ! \Gamma(-\alpha-i+j+1)} r^{-j}(-s)^{j-k} u_{i} & \text { if } 0 \leq k \leq n, \\
0 & \text { if } k>n .\end{cases}
$$

Therefore $u x=\left(u_{k} x_{k}\right) \in c s$ whenever $x \in b_{0}^{r, s}\left(\nabla^{(\alpha)}\right)$ if and only if $U^{r, s} y \in c$ whenever $y \in c_{0}$. This implies that $u=\left(u_{k}\right) \in\left[b_{0}^{r, s}\left(\nabla^{(\alpha)}\right)\right]^{\beta}$ if and only if $U^{r, s} \in\left(c_{0}: c\right)$. We obtain by part (ii) of Lemma 3.1 that $\left[b_{0}^{r, s}\left(\nabla^{(\alpha)}\right)\right]^{\beta}=U_{2}^{r, s} \cap U_{3}^{r, s}$. The proof can be completed in a similar way by parts (iii), (iv), (v) instead of Part (ii) of Lemma 3.1, so we omit the details.

Theorem 3.4 The spaces $\left[b_{0}^{r, s}\left(\nabla^{(\alpha)}\right)\right]^{*}$ and $\left[b_{c}^{r, s}\left(\nabla^{(\alpha)}\right)\right]^{*}$ are equivalent to $\ell_{1}$.

Proof Proof follows from Theorem 3.6 of Bişgin [11] and the fact that if $Z$ is a Banach space, then $\left[Z\left(\nabla^{(\alpha)}\right)\right]^{*}$ is equivalent to $X^{*}[17]$.

\section{Conclusion}

In this paper, we have discussed some results obtained from the matrix domain of the binomial matrix and the difference matrix of fractional order. Our main aim is to generalize the results on the matrix domain of the Euler matrix. It is immediate that our results reduce to the sequence spaces defined in $[11,12,18,23,25,26]$.

\section{Acknowledgements}

We wish to thank the referee for his/her constructive comments and suggestions.

Funding

This work is supported by NSF of China (11371289).

Competing interests

The authors declare that they have no competing interests.

Authors' contributions

Both authors contributed equally to the writing of the paper. All authors read and approved the final manuscript.

\section{Publisher's Note}

Springer Nature remains neutral with regard to jurisdictional claims in published maps and institutional affiliations.

Received: 6 July 2018 Accepted: 1 October 2018 Published online: 10 October 2018

\section{References}

1. Altay, B., Başar, F:: On some Euler sequence spaces of nonabsolute type. Ukr. Math. J. 57, 1-17 (2005)

2. Altay, B., Başar, F., Mursaleen, M.: On the Euler sequence spaces which include the spaces $\ell_{p}$ and $\ell_{\infty}$ I. Inf. Sci. 176, 1450-1462 (2006)

3. Baliarsingh, P.: On a fractional difference operator. Alex. Eng. J. 55, 1811-1816 (2016) 
4. Baliarsingh, P., Dutta, S.: On the classes of fractional order difference sequence spaces and their matrix transformation. Appl. Math. Comput. 250, 665-674 (2015)

5. Baliarsingh, P., Dutta, S.: A unifying approach to the difference operators and their applications. Bol. Soc. Parana. Mat. 33, 49-57 (2015)

6. Başarir, M., Kara, E.E.: On compact operators on the Riesz $B^{m}$-difference sequence spaces. Iran. J. Sci. Technol. 35 , 279-285 (2011)

7. Başarir, M., Kara, E.E.: On some difference sequence spaces of weighted means and compact operators. Ann. Funct. Anal. 2, 114-129 (2011)

8. Başarir, M., Kara, E.E.: On compact operators on the Riesz $B^{m}$-difference sequence spaces II. Iran. J. Sci. Technol. 33, 371-376 (2012)

9. Başarir, M., Kara, E.E.: On the B-difference sequence space derived by generalized weighted mean and compact operators. J. Math. Anal. Appl. 391, 67-81 (2012)

10. Başarir, M., Kara, E.E.: On the $m$ th order difference sequence space of generalized weighted mean and compact operators. Acta Math. Sci. 33, 797-813 (2013)

11. Bişgin, M.C.: The binomial sequence spaces of nonabsolute type. J. Inequal. Appl. 2016, 309 (2016)

12. Bişgin, M.C.: The binomial sequence spaces which include the spaces $\ell_{p}$ and $\ell_{\infty}$ and geometric properties. J. Inequal. Appl. 2016, 304 (2016)

13. Choudhary, B., Nanda, S.: Functional Analysis with Application. Wiley, New Delhi (1989)

14. Çolak, R., Et, M.: On some generalized difference sequence spaces and related matrix transformations. Hokkaido Math. J. 26, 483-492 (1997)

15. Et, M.: On some topological properties of generalized difference sequence spaces. Int. J. Math. Math. Sci. 24, 785-791 (2000)

16. Et, M., Colak, R.: On generalized difference sequence spaces. Soochow J. Math. 21, 377-386 (1995)

17. Et, M., Nuray, F.: $\Delta^{m}$-statistical convergence. Indian J. Pure Appl. Math. 32, 961-969 (2001)

18. Kadak, U., Baliarsingh, P.: On certain Euler difference sequence spaces of fractional order and related dual properties. J. Nonlinear Sci. Appl. 8, 997-1004 (2015)

19. Kara, E.E.: Some topological and geometrical properties of new Banach sequence spaces. J. Inequal. Appl. 2013, 38 (2013)

20. Kara, E.E., Illkhan, M.: On some Banach sequence spaces derived by a new band matrix. Br. J. Math. Comput. Sci. 9, 141-159 (2015)

21. Kara, E.E., Illkhan, M.: Some properties of generalized Fibonacci sequence spaces. Linear Multilinear Algebra 64, 2208-2223 (2016)

22. Kizmaz, H.: On certain sequence spaces. Can. Math. Bull. 24, 169-176 (1981)

23. Meng, J., Song, M.M.: Binomial difference sequence spaces of order m. Adv. Differ. Equ. 2017, 241 (2017)

24. Meng, J., Song, M.M.: On some binomial $B^{(m)}$-difference sequence spaces. J. Inequal. Appl. 2017, 194 (2017)

25. Meng, J., Song, M.M.: On some binomial difference sequence spaces. Kyungpook Math. J. 57, 631-640 (2017)

26. Polat, H., Başar, F.: Some Euler spaces of difference sequences of order $m$. Acta Math. Sci. 27, 254-266 (2007)

27. Song, M.M., Meng, J.: Some normed binomial difference sequence spaces related to the $\boldsymbol{\ell}_{p}$ spaces. J. Inequal. Appl. $2017,128(2017)$

28. Stieglitz, M., Tietz, H.: Matrixtransformationen von folgenräumen eine ergebnisubersict. Math. Z. 154, 1-16 (1977)

29. Wilansky, A.: Summability Through Functional Analysis. North-Holland Mathematics Studies, vol. 85. Elsevier, Amsterdam (1984)

\section{Submit your manuscript to a SpringerOpen ${ }^{\circ}$ journal and benefit from:}

- Convenient online submission

- Rigorous peer review

- Open access: articles freely available online

- High visibility within the field

- Retaining the copyright to your article

Submit your next manuscript at $\boldsymbol{~ s p r i n g e r o p e n . c o m ~}$ 